\title{
Synthesis, Characterization and Antimicrobial Evaluation of Novel (E)-N'-(4-(1-((3,4-dimethoxypyridin-2-yl)methyl)-1H- 1,2,3-triazol-4-yl)benzylidene)benzohydrazide Derivatives
}

\author{
SWAMY SAIDUGARI ${ }^{1,2 *}$, LAKSHMANA RAO VADALI ${ }^{1}, \mathrm{~K}$ VIDYA $^{2}$ and B RAM ${ }^{3}$ \\ 'Mylan Laboratories Limited, Bollaram, Jinnaram (Mandal), \\ Medak (Dist), Hyderabad, Telangana State, India. \\ 2JNTU-H College of Engineering, Nachupally (Kondagattu), \\ Kodimyalmandal, Jagityal, Karimnagar -505 501, Telengana State, India. \\ ${ }^{3}$ Green Evolution Laboratories, Wangapally Village, Telangana State, 500085, India. \\ *Corresponding author E-mail:swamysaidugari2015@gmail.com
}

http://dx.doi.org/10.13005/ojc/320445

(Received: May 30, 2016; Accepted: August 02, 2016)

\begin{abstract}
The synthesis of novel 1,2,3-triazole-hydrazone derivatives embedded with 3,4-dimethoxy pyridine ring nucleus is described. These derivatives were prepared utilizing, 2-(chloromethyl)-3,4dimethoxypyridine 1, 4-ethynylbenzaldehye 5 and various benzohydrazides7a-7j. The structures of the newly synthesized 1,2,3-triazole-hydrazones $8 \mathrm{a}$-j was established on the basis of the spectroscopic techniques like ${ }^{1} \mathrm{H}$ NMR, mass and IR data. They were evaluated against a panel of bacterial and fungal pathogens such as Staphylococcus. pyogens, Staphylococcus. Aureus (Gram positive bacteria), Escherichia.coli, Pseudomonas. aeruginosa (Gram negative bacteria) and Aspergillus niger and Candida albicans (Fungal stains). Compounds 8b, 8c, 8d, 8e and $8 \mathrm{f}$ with $\mathrm{R}=4-\mathrm{OH}, 4-\mathrm{OMe}, 4-\mathrm{SO}_{2} \mathrm{Me}$, $3,45,-\mathrm{OMe}$ and $3-\mathrm{NO}_{2}$ respectively showed moderate antibacterial activity while compounds $8 \mathrm{~b}, 8 \mathrm{~d}$, $8 \mathrm{i}$ and $8 \mathrm{j}$ with $\mathrm{R}=4-\mathrm{OH}, 4-\mathrm{SO}_{2} \mathrm{Me}, 3,5$-dichloro and 2,5-difluoro substitution exhibited very good fungal activity.
\end{abstract}

Keywords: 1,2,3-triazole, Hydrazone, 4-ethynylbenzaldehyde, Synthesis, Antifungal activity.

\section{INTRODUCTION}

The growth of 1,2,3-triazoles for drug discovery and industrial use has been shown to be very resourceful.The interest in the 1,2,3-triazole is due to it being non-toxic, benign and stable. Triazoles are predominantly attractive for medicinal use because they are more expected to be water soluble than normal aromatic compounds, and are stable in biological systems ${ }^{1}$. These triazole products readily connect with biological targets, through hydrogen bonding and dipole interactions ${ }^{2}$. Some of the pharmaceutically prominent triazole antifungal drugs are fluconazole ${ }^{3}$, isavuconazole ${ }^{4}$, itraconazole ${ }^{5}$, 
voriconazole ${ }^{6}$, pramiconazole $e^{7}$, and posaconazole ${ }^{8}$. Also, the examples of triazole crop protection fungicides includesepoxiconazole ${ }^{9}$, triadimenol $^{10}$, propiconazole ${ }^{11}$, metconazole ${ }^{12}$, cyproconazole ${ }^{13}$, tebuconazole ${ }^{14}$, flusilazole ${ }^{15}$ and paclobutrazol ${ }^{16}$. Derivatives of 1,2,3-triazole have been found to have anti-HIV ${ }^{17}$, anti-allergenic ${ }^{18}$, cytostatic ${ }^{19}$, virostatic ${ }^{20}$, anti-inflammatory ${ }^{21}$ activities, furthermore, these triazoles are also being studied for the treatment of obesity ${ }^{22}$ and anti-oxidant activity ${ }^{23}$.

Hydrazone derivatives of heteroaromatic compounds have also been reported to possess anti-inflammatory ${ }^{24,25}$, anticancer ${ }^{26}$, antitumor ${ }^{27}$, antibacterial or plant-growth activity ${ }^{28,29}$.

The frequency of bacterial and fungal infections is an important contemporary problem due to the emerging new infectious diseases and increasing multi-drug resistance of microbial pathogens ${ }^{30}$. The widespread use of antibiotics has contributed to the growing infection rate since fungal infections occur after antibiotic therapy, which has the effect of killing the beneficial bacteria that normally suppress fungi. The development of new effective antifungal and antibacterial agents is strongly needed. Herein, we report the synthesis and antimicrobial evaluation of new $1,2,3$ triazole derivatives linked with pyridine and hydrazone fragments. The structures of the synthesized compounds were determined by $1 \mathrm{H}$ NMR, mass and IR spectroscopy.

\section{RESULTS AND DISCUSSION}

\section{Chemistry}

The synthesis of new $(E)-\mathrm{N}^{\prime}-(4-(1-((3,4-$ dimethoxypyridin-2-yl)methyl)-1H-1,2,3-triazol-4-yl) benzylidene)benzohydrazide derivatives is illustrated in Scheme-1. The reaction of 2-(chloromethyl)-3,4dimethoxypyridine ${ }^{1}$ with sodium azide in DMF at $90^{\circ} \mathrm{C}$ afforded azide derivative ${ }^{2}$. Copper(I)-catalyzed, Huisgen [3+2] cycloaddition reaction ${ }^{31-32}$ of azide ${ }^{2}$ with 4-ethynylbenzaldehyde ${ }^{5}$ in acetonitrile at reflux produced 4-(1-((3,4-dimethoxypyridin-2-yl)methyl)$1 \mathrm{H}-1,2,3$-triazol-4-yl)benzaldehyde 6 in $95 \%$ yield. Condensation of aldehyde ${ }^{6}$ with benzohydrazides $7 a-7 j$ in ethanol resulted in the formation of hydrazonederivatives $8 \mathrm{a}-\mathrm{j}$ in quantitative yields. The synthesis 4-ethynylbenzaldehyde ${ }^{5}$ is achieved as follows, reaction of 4-iodobenzaldehyde ${ }^{3}$ with TMS- acetylene in presence of $\mathrm{Pd}\left(\mathrm{PPh}_{3}\right)_{2} \mathrm{Cl}_{2}, \mathrm{Cul}, \mathrm{Et}_{3} \mathrm{~N}$ in THF gave the corresponding trimethylatedsillyl derivative $e^{4}$. De-protection of the trimethylsillyl group in presence of $\mathrm{K}_{2} \mathrm{CO}_{3}$ in methanol resulted in the desired benzaldehyde ${ }^{5}$.

The structures of the synthesized compounds were confirmed by ${ }^{1} \mathrm{H}$ NMR, Mass and IR data. As a representative example, the ${ }^{1} \mathrm{H}$ NMR spectra of $(E)-\mathrm{N}^{\prime}-(4-(1-((3,4-d i m e t h o x y p y r i d i n-2-$ yl)methyl)-1H-1,2,3-triazol-4-yl)benzylidene)-4methoxybenzohydrazide is as follows, the protons resonating at $11.78 \mathrm{ppm}$ and $8.48 \mathrm{ppm}$ as broad singlet corresponds to the characteristic $-\mathrm{CONH}$ and $-\mathrm{CH}=\mathrm{N}$ - respectively, while the protons resonating at $8.72 \mathrm{ppm}$ (pyridine ring proton) and $7.23 \mathrm{ppm}$ (overlap of triazole and pyridine ring proton signal), is assigned to triazole ring and pyridine ring protons. The multiplet at 7.94-7.91 ppm (four proton integration), a broad singlet at $7.81 \mathrm{ppm}$ (two proton integration) and a doublet at $7.07 \mathrm{ppm}$ (two proton integration is assigned to the protons of the 4-methoxy phenyl ring and the phenyl ring flanked to triazole ring. The aliphatic protons are observed in the expected region. The mass and IR spectral data of the hydrazone compounds are in agreement with the desired structures.

\section{Antibacterial and antifungal activity}

The screening results of $(E)-\mathrm{N}^{\prime}-(4-(1-((3,4-$ dimethoxypyridin-2-yl)methyl)-1H-1,2,3-triazol-4-yl) benzylidene)benzohydrazide derivatives $8 \mathrm{a}-\mathrm{j}$ are tabulated in table 1. From table-1 it is observed that hydrazone derivatives $8 \mathrm{~b}, 8 \mathrm{c}, 8 \mathrm{~d}, 8 \mathrm{e}$ and $8 \mathrm{f}$ with $\mathrm{R}=4-\mathrm{OH}, 4-\mathrm{OMe}, 4-\mathrm{SO}_{2} \mathrm{Me}, 3,4,5,-\mathrm{OMe}$ and $3-\mathrm{NO}_{2}$ respectively showed moderate antibacterial activity while the hydrazone derivatives $8 \mathrm{a}, 8 \mathrm{i}$ and $8 \mathrm{j}$ with $\mathrm{R}=\mathrm{H}, 3,5$-dichloro and 2,5-difluoro showed weak antibacterial against both Gram positive and Gram negative bacteria with reference to ciprofloxacin as the standard drug.

In case of fungal screening evaluation, hydrazone derivatives $8 b, 8 d, 8 i$ and $8 j$ with $R=4-O H$, 4- $\mathrm{SO}_{2} \mathrm{Me}, 3,5$-dichloro and 2,5-difluoro substitution exhibited very good fungal activity and the remaining hydrazone derivatives in the series showed moderate antifungal activity against the tested fungal strains with reference to Greseofluvin as the standard drug. 


\section{EXPERIMENTAL}

The solvents were purified according to standard procedures prior to use, and all commercial chemicals were used as received. For thin-layer chromatography (TLC) analysis, Merck pre-coated plates (silica gel 60 F254) were used and spots were visualized with UV light. Merck silica gel 60 (230-400 mesh) was used for flash column chromatography and the eluting solvents are indicated in the procedures. Melting point (m.p.) determinations were performed by using Mel-temp apparatus and are uncorrected. ${ }^{1} \mathrm{H}$ NMR spectra were recorded in Varian MR-400 MHz instrument. Chemical shifts are reported in $\delta \mathrm{ppm}$ (parts per million) downfield from tetramethylsilane (TMS) with reference to internal standard and the signals were reported as $s$ (singlet), $d$ (doublet), dd (doblet of doblet), $t$ (triplet), $q$ (quartet), $m$ (multiplet) and coupling constants are in $\mathrm{Hz}$. The mass spectra were recorded on Agilent ion trap MS. Infrared (IR) spectra were recorded on a Perkin Elmer FT-IR spectrometer.

\section{2-(azidomethyl)-3,4-dimethoxypyridine 2}

Sodium azide $(0.365 \mathrm{~g}, 5.60 \mathrm{mmol})$ was added to a solution of compound $1(1 \mathrm{~g}, 5.33 \mathrm{mmol})$ in DMF (5 mL) at room temperature. The reaction mixture was heated to $100^{\circ} \mathrm{C}$ for $1 \mathrm{~h}$. After completion of the reaction (by T.L.C), the reaction contents were cooled to room temperature and diluted with ethylacetate $(15 \mathrm{~mL})$ and water $(5 \mathrm{~mL})$. The organic layer was separated and washed with brine solution (2X $5 \mathrm{~mL})$, separated, dried over anhydrous sodium sulphate, filtered and concentrated under reduced pressure to obtain the crude compound 2 . The crude compound was utilized in the next step without further purification.

\section{4-(2-(trimethylsilyl)ethynyl)benzaldehyde 4}

To a solution of 4-iodobenzaldehyde 3 $(1 \mathrm{~g}, 4.30 \mathrm{mmol})$ in tetrahydrofuran $(12 \mathrm{~mL})$, in a seal tube, were added sequentially, copper(I) iodide $(0.14 \mathrm{mmol})$, triphenylphosphine $(0.39 \mathrm{mmol})$, bis(triphenylphosphine)palladium(II) dichloride $(0.14 \mathrm{mmol})$ and trimethylsilylacetylene $(0.59 \mathrm{~mL}$, $4.14 \mathrm{mmol}$ ) under argon atmosphere. After string<smiles>C#Cc1ccc(C=O)cc1</smiles><smiles>[R]c1ccc(C(=O)NN=NC(=O)c2ccccc2)cc1/C=N\NC(=O)c1ccc(-c2cn(Cc3nccc(OC)c3OC)nn2)cc1</smiles>

$8 \mathbf{a}-8 \mathbf{j}$

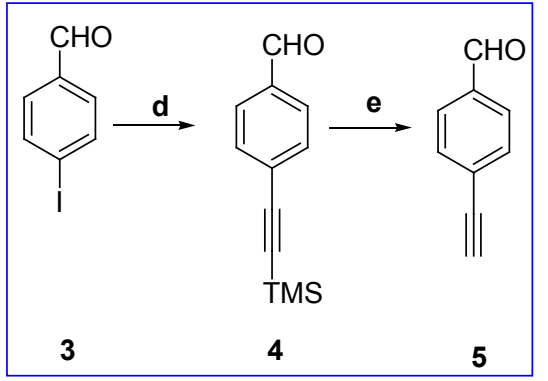

\begin{tabular}{|ll|}
\multicolumn{1}{c}{$8 \mathrm{a}-8 \mathrm{j}$} \\
\hline 8a. $\mathrm{R}=\mathrm{H}$ & 8f. $\mathrm{R}=3-\mathrm{NO}_{2}$ \\
8b. $\mathrm{R}=4-\mathrm{OH}$ & $8 \mathrm{~g} \cdot \mathrm{R}=4-\mathrm{Br}$ \\
8c. $\mathrm{R}=4-\mathrm{OMe}$ & $8 \mathrm{~h} . \mathrm{R}=4-\mathrm{Cl}$ \\
8d. $\mathrm{R}=4-\mathrm{SO}_{2} \mathrm{Me}$ & $8 \mathrm{i} . \mathrm{R}=3,5$-dichloro \\
8e. $\mathrm{R}=3,4,5-\mathrm{OMe}$ & 8j. $\mathrm{R}=2,5$-difluoro \\
\hline
\end{tabular}

Reaction conditions:a) $\mathrm{NaN}_{3}$, DMF, $90^{\circ} \mathrm{C}$; b) Cul, acetonitrile, reflux, 1h; c) Benzohydrazides7a-j, ethanol, reflux, $1 \mathrm{~h}$; d) Bis(triphenylphosphine)palladium(II) dichloride, Trimethylacetylene, Cul, Triphenylphosphine, Triethylamine, THF, $65^{\circ} \mathrm{C}, 2 \mathrm{~h}$; e) potassium carbonate, Methanol, room temperature, $1 \mathrm{~h}$; 
for $15 \mathrm{~min}$, triethylamine (12.9 mmol) was added and the resultant mixture was heated at 65 úc for $30 \mathrm{~min}$. The reaction contents were diluted with ethyl acetate $(15 \mathrm{~mL})$, washed with water $(2 \times 15 \mathrm{~mL})$, washed with brine solution $(10 \mathrm{~mL})$. The organic layer was separated and dried over $\mathrm{Na}_{2} \mathrm{SO}_{4}$. The solvent was evaporated under reduced pressure to obtain compound 4 as a viscous pale yellow oily liquid. The crude compound was utilized in the next step without further purification.

\section{4-ethynylbenzaldehyde 5}

A mixture of 4-((trimethylsilyl)ethynyl) benzaldehyde 4 (1g, $4.94 \mathrm{mmol})$ and potassium carbonate $(1 \mathrm{~g}, 7.41 \mathrm{mmol})$ were stirred in methanol (25 mL) at room temperature for 1 hour. The reaction mixture was diluted with ethylacetate $(20 \mathrm{~mL})$ and washed with water $(2 \times 20 \mathrm{~mL})$ followed by brine solution. The organic solvent was concentrated under reduced pressure to obtain compound 5. Yellow solid; Yield: $0.29 \mathrm{~g}$ (90\%); M.p.: 84-86 ${ }^{\circ} \mathrm{C}$; IR (KBr): $\mathrm{U}_{\max } 3454,329,3220,2836,2784,2739,2098,1930$, $1699,1686,1601,1560,1408,1386,1364,1303$, 1286, 1206, 1163, 1102, 1012, 975, 954, 924, 844, 829, 739, 679, 581, 530, $508 \mathrm{~cm}^{-1} ; 1 \mathrm{H}$ NMR (400 MHz, $\left.\mathrm{CDCl}_{3}\right): \delta 3.30(1 \mathrm{H}, \mathrm{s}), 7.66(\mathrm{~d}, \mathrm{~J}=8.20 \mathrm{~Hz}, 2 \mathrm{H})$, $7.86(\mathrm{~d}, \mathrm{~J}=8.20 \mathrm{~Hz}, 2 \mathrm{H}), 10.02(\mathrm{~s}, 1 \mathrm{H})$.
4-(1-((3,4-dimethoxypyridin-2-yl)methyl)-1H-1,2,3triazol-4-yl)benzaldehyde 6

4-ethynylbenzaldehyde 5 (1g, $7.68 \mathrm{~mol})$ was added to a solution of compound $3(0.67 \mathrm{~g}$, $5.15 \mathrm{mmol})$ in acetonitrile $(10 \mathrm{~mL})$ followed by the addition of Cul(10 mol \%). The reaction mixture was heated to reflux for $1 \mathrm{~h}$. After completion of the reaction (monitored by T.L.C), the reaction mixture was diluted with ethylacetate $(20 \mathrm{~mL})$ and water $(20 \mathrm{~mL})$. The organic layer was washed with briene solution, separated and dried over $\mathrm{Na}_{2} \mathrm{SO}_{4}$, filtered and concentrated under reduced pressure to obtain 6. Yellow solid; Yield: $90 \%$; M.p.: $131-132^{\circ} \mathrm{C}$; IR (KBr): $U_{\max } 3351,3130,3100,3047,3017,2986,2945$, 2841, 2722, 2604, 1696, 1608, 1589, 1494, 1452, 1429, 1400, 1349, 1304, 1270, 1232, 1210, 1171, 1144, 1103, 1069, 1046, 996, 971, 947, 907, 832, $755,728,688,657,626,553,515,486,463 \mathrm{~cm}^{-1} ;{ }^{1} \mathrm{H}$ NMR (400 MHz, DMSO-d $): \delta 10.0(\mathrm{~s}, 1 \mathrm{H}), 8.78$ (brs, $1 \mathrm{H}), 8.18$ (brs, $1 \mathrm{H}), 8.10$ (d, J = 7.8 Hz, 2H), 7.94 (d, $\mathrm{J}=7.8 \mathrm{~Hz}, 2 \mathrm{H}$ ), 7.10 (brs, $1 \mathrm{H}), 5.78$ (s, 2H), 3.96 (s, $3 \mathrm{H}), 3.78$ (s, 3H); ESI- MS: m/z, 324.9(M+1).

\section{Experimental procedure for synthesis of} benzohydrazides7a-j [34-35]

Benzoic acids a-j ( $8.12 \mathrm{mmol})$ was dissolved in ethanol $(15 \mathrm{~mL})$ and added catalytic qty of conc. $\mathrm{H}_{2} \mathrm{SO}_{4}$ and heated to reflux for $10 \mathrm{~h}$. Ethanol was

Table 1: Results of Antibacterial and Antifungal activity of Compounds 8a-j

\begin{tabular}{|c|c|c|c|c|c|c|}
\hline \multirow[t]{3}{*}{ Compound no. } & \multicolumn{2}{|c|}{ Gramnegative bacteria } & \multicolumn{2}{|c|}{ Gram positive bacteria } & \multirow{3}{*}{ A. niger } & \multirow{3}{*}{$\begin{array}{l}\text { Fungi } \\
\text { C. albicans }\end{array}$} \\
\hline & E. coli & P.aeruginosa & S.pyogens & S.aureus & & \\
\hline & \multicolumn{4}{|c|}{ Zone of inhibition expressed in $\mathrm{mm}$} & & \\
\hline $8 a$ & 12 & 11 & 9 & 10 & 20 & 18 \\
\hline $8 b$ & 17 & 18 & 14 & 16 & 26 & 22 \\
\hline $8 c$ & 16 & 17 & 12 & 14 & 22 & 17 \\
\hline $8 d$ & 20 & 21 & 18 & 19 & 25 & 20 \\
\hline $8 e$ & 20 & 22 & 17 & 17 & 19 & 16 \\
\hline $8 f$ & 19 & 20 & 18 & 18 & 20 & 18 \\
\hline $8 g$ & - & - & - & - & 21 & 19 \\
\hline $8 \mathrm{~h}$ & - & - & - & - & 19 & 17 \\
\hline $8 i$ & 12 & 11 & 9 & 10 & 26 & 22 \\
\hline $8 \mathrm{j}$ & 11 & 13 & 12 & 11 & 25 & 23 \\
\hline Ciprofloxacin & 28 & 27 & 22 & 22 & & \\
\hline Greseofluvin & & 28 & 24 & & & \\
\hline
\end{tabular}

Standard drug concentration: $250 \mu \mathrm{g} / \mathrm{mL}$; “___ No activity 
evaporated and the obtained residue was diluted with ethylacetate $(25 \mathrm{~mL})$. The organic layer was washed with aqueous saturated $\mathrm{NaHCO}_{3}(3 \times 15$ $\mathrm{mL})$ followed by water $(2 \times 15 \mathrm{~mL})$ and brine solution $(20 \mathrm{~mL})$. The organic layer was separated, dried over sodium sulphate, filtered and evaporated to obtain respective ethyl benzoates.

To the above prepared respective ethyl benzoates $(6.65 \mathrm{mmol})$ in ethanol was added hydrazine-hydrate $(40.0 \mathrm{mmol})$ and refluxed for $8 \mathrm{~h}$. Ethanol was evaporated from the reaction mixture and the precipitated solids were slurred with petether (5 times) and filtered at the pump and dried to obtain benzohydrazides7a-7j.

\section{General procedure for the synthesis of hydrazone derivatives $\mathbf{8 a - 8} \mathbf{j}$}

To a solution of compound 6 (100 mg, $0.308 \mathrm{mmol}$ ) in ethanol was added corresponding benzohydrazides $7 a-7 j$ ( $0.308 \mathrm{mmol})$ and heated to reflux for $30 \mathrm{~min}$. The precipitated solids were filtered at the pump and dried to afford hydrazone derivatives $\mathbf{8 a - 8 j}$ in quantitative yields.

(E)-N'-(4-(1-((3,4-dimethoxypyridin-2-yl) methyl)-1 H-1,2,3-triazol-4-yl)benzylidene) benzohydrazide $8 \mathbf{a}$

Pale yellow solid; Yield: 90\%; M.p.: 101$102^{\circ} \mathrm{C} ; \mathrm{IR}(\mathrm{KBr}): \mathrm{U}_{\max } 3425,3260,3138,3057,3023$, 2974, 2944, 2842, 1655, 1584, 1538, 1489, 1456, 1426, 1358, 1299, 1278, 1228, 1177, 1139, 1101, 1066, 997, 972, 947, 914, 821, 804, 762, 718, 626, $534,493 \mathrm{~cm}^{-1} ;{ }^{1} \mathrm{H}$ NMR (400 MHz, DMSO-d 6 ): $\delta 11.89$ (brs, 1H), 8.66 (s, 1H), 8.44 (s, 1H), 8.13 (brs, 1H), 7.99-7.92 (m, 4H), 7.80 (d, J = 10.8 Hz, 2H), 7.637.52 (m, 3H), 7.16 (brs, 1H), 5.72 (brs, 2H), 3.91 (s, $3 \mathrm{H}), 3.81$ (s, 3H); ESI -MS: m/z, $443.0(\mathrm{M}+1)$.

(E)-N'-(4-(1-((3,4-dimethoxypyridin-2-yl) methyl)-1 H-1,2,3-triazol-4-yl)benzylidene)-4hydroxybenzohydrazide $\mathbf{8 b}$

Pale yellow solid; Yield: 88\%; M.p.: 128$129^{\circ} \mathrm{C}$; IR (KBr): U $\mathrm{max} 3270,3147,3020,2944,2837$, 2792, 2672, 2601, 1907, 1645, 1607, 1588, 1542, $1508,1492,1450,1358,1301,1281,1237,1173$, 1143, 1105, 1072, 995, 972, 951, 912, 838, 809, 761, 721, 685, 622, 598, $507 \mathrm{~cm}^{-1} ;{ }^{1} \mathrm{H}$ NMR (400 $\mathrm{MHz}, \mathrm{DMSO}-\mathrm{d}_{6}$ ): $\delta 11.68$ (brs, $\left.1 \mathrm{H}\right), 10.13$ (brs, $1 \mathrm{H}$ ), 8.66 (brs, 1H), 8.44 (brs, 1H), 8.10 (d, J = 9.6 Hz,
2H), 7.96-7.80 (m, 5H), 7.15 (brs, 1H), 6.86 (d, J = $10.6 \mathrm{~Hz}, 2 \mathrm{H}), 5.72$ (s, 2H), 3.90 (s, 3H), 3.80 (s, 3H); ESI- MS: m/z, $459.0(\mathrm{M}+1)$.

(E)-N'-(4-(1-((3,4-dimethoxypyridin-2-yl) methyl)-1 H-1,2,3-triazol-4-yl)benzylidene)-4methoxybenzohydrazide $8 \mathrm{c}$

Off white solid; Yield: $84 \%$; M.p.: $96-97^{\circ} \mathrm{C}$;IR $(\mathrm{KBr}): U_{\max } 3570,3527,3453,3409,3366,3288$, 3158, 3093, 2939, 2836, 1647, 1603, 1539, 1498, 1456, 1426, 1355, 1302, 1256, 1179, 1143, 1109 , 1068, 1025, 988, 915, 837, 760, 725, 689, 614, 567, $537,509,455 \mathrm{~cm}^{-1}$; ${ }^{1} \mathrm{H}$ NMR (400 MHz, DMSO-d 6 ): $\delta 11.78$ (brs, $1 \mathrm{H},-\mathrm{CONH}), 8.72$ (brs, $1 \mathrm{H}), 8.48$ (brs, $1 \mathrm{H},-\mathrm{CH}=\mathrm{N}-)$, 8.13 (brs, $1 \mathrm{H}), 7.94-7.91(\mathrm{~m}, 4 \mathrm{H}), 7.81$ (brs, 2H), 7.23 (brs, $1 \mathrm{H}$, triazole), 7.07 (d, J = 10.4 $\mathrm{Hz}, 2 \mathrm{H}$, ), 5.72 (brs, 2H), 3.91 (s, 3H), 3.84 (s, 3H), 3.81 (s, 3H); ESI- MS: m/z, 443.0(M+1).

(E)-N'-(4-(1-((3,4-dimethoxypyridin-2-yl) methyl)-1H-1,2,3-triazol-4-yl)benzylidene)-4(methylsulfonyl)benzohydrazide 8d

Yellow solid; Yield: $82 \%$; M.p.: $134-135^{\circ} \mathrm{C}$; IR (KBr): $U_{\max } 3570,3527,3453,3409,3366,3288$, 3158, 3093, 2939, 2836, 1647, 1603, 1539, 1498, 1456, 1426, 1355, 1302, 1256, 1179, 1143, 1109 , 1068, 1025, 988, 915, 837, 760, 725, 689, 614, 567, 537, 509, $455 \mathrm{~cm}^{-1}$; ${ }^{1} \mathrm{H}$ NMR (400 MHz, DMSO$\left.d_{6}\right): \delta 12.11$ (brs, $\left.1 \mathrm{H}\right), 8.67$ (brs, $\left.1 \mathrm{H}\right), 8.48(\mathrm{~s}, 1 \mathrm{H})$, 8.17-8.02 (m, 5H), 7.97 (d, J = 10.8 Hz, 2H), 7.89 (d, J = $10.8 \mathrm{~Hz}, 2 \mathrm{H}), 7.15$ (brs, 1H), 5.72 (brs, 2H), 3.90 (s, 3H), 3.80 (s, 3H), 3.30 (s, 3H); ESI- MS: $\mathrm{m} / \mathrm{z}, 521.0(\mathrm{M}+1)$.

(E)-N'-(4-(1-((3,4-dimethoxypyridin-2-yl) methyl)-1H-1,2,3-triazol-4-yl)benzylidene)-3,4,5trimethoxybenzohydrazide $8 \mathrm{e}$

Pale yellow solid; Yield: 84\%; M.p.: 89$90^{\circ} \mathrm{C} ;{ }^{1} \mathrm{H}$ NMR $\left(400 \mathrm{MHz}\right.$, DMSO- $\left.\mathrm{d}_{6}\right): \delta 11.74$ (brs, $1 \mathrm{H}), 8.65(\mathrm{~s}, 1 \mathrm{H}), 8.48(\mathrm{~s}, 1 \mathrm{H}), 7.96(\mathrm{~d}, \mathrm{~J}=10.8 \mathrm{~Hz}$, $2 \mathrm{H}), 7.80$ (d, J = 10.8 Hz, 2H), 7.24 (s, 2H), 5.72 (brs, 2H), 3.90 (s, 3H), 3.89 (s, 3H); ESI MS: m/z, 533.0 $(\mathrm{M}+1)$.

(E)-N'-(4-(1-((3,4-dimethoxypyridin-2-yl) methyl)-1 H-1,2,3-triazol-4-yl)benzylidene)-3nitrobenzohydrazide $8 f$

Yellow solid; Yield: $92 \%$; M.p.: $84-85^{\circ} \mathrm{C}$; ${ }^{1} \mathrm{H}$ NMR (400 MHz, DMSO-d $)$ : $\delta 11.20$ (brs, $1 \mathrm{H}$ ), 10.16 (brs, 1H), 8.77 (brs,1H), 8.60 (brs, 1H), 8.44 
(d, J = 9.8 Hz, 2H), 8.26 (d, J = 9.6 Hz, 2H), 7.70$7.62(\mathrm{~m}, 3 \mathrm{H}), 5.72$ (brs, 2H), 3.90 (s, 3H), 3.80 (s, $3 \mathrm{H})$; ESI -MS: m/z, $488.0(\mathrm{M}+1)$.

(E)-N'-(4-(1-((3,4-dimethoxypyridin-2-yl) methyl)-1H-1,2,3-triazol-4-yl)benzylidene)-4bromobenzohydrazide $8 \mathrm{~g}$

Yellow solid; Yield: $83 \%$; M.p.: $129-130^{\circ} \mathrm{C}$;IR $(\mathrm{KBr}): \mathrm{U}_{\max } 3460,3228,3070,2986,2941,2838$, $1651,1588,1552,1489,1462,1446,1425,1395$, $1366,1349,1301,1270,1229,1180,1145,1104$, $1065,1004,974,949,914,882,839,822,753,709$, $675,623,597,572,535,504,458 \mathrm{~cm}^{-1} ;{ }^{1} \mathrm{H}$ NMR (400 $\mathrm{MHz}, \mathrm{DMSO}-\mathrm{d}_{6}$ ): $\delta 11.95$ (brs, $\left.1 \mathrm{H}\right), 8.67$ (brs, $\left.1 \mathrm{H}\right)$, 8.46 (brs, $1 \mathrm{H}$ ), 8.12 (brs, $1 \mathrm{H}$ ), 7.97 (d, J = $10.8 \mathrm{~Hz}$, $2 \mathrm{H}), 7.88(\mathrm{~d}, \mathrm{~J}=11.2 \mathrm{~Hz}, 2 \mathrm{H}), 7.81-7.74(\mathrm{~m}, 4 \mathrm{H})$, 7.16 (brs, $1 \mathrm{H}$ ), 5.72 (brs, 2H), 3.90 (s, 3H), 3.80 (s, $3 \mathrm{H})$; ESI-MS: m/z, $521.0(\mathrm{M}+1)$.

(E)-N'-(4-(1-((3,4-dimethoxypyridin-2-yl) methyl)-1H-1,2,3-triazol-4-yl)benzylidene)-4chlorobenzohydrazide $8 \mathrm{~h}$

Pale yellow solid; Yield: $86 \%$; M.p.: $128-$ $129^{\circ} \mathrm{C}$; IR (KBr): $\mathrm{U}_{\max } 3744,3461,3230,3089,2985$, 2942, 2838, 1655, 1592, 1566, 1548, 1490, 1457, $1427,1358,1302,1272,1237,1180,1144,1094$, $1065,1012,977,946,915,842,752,728,677,602$, $534,495 \mathrm{~cm}^{-1} ;{ }^{1} \mathrm{H}$ NMR $(400 \mathrm{MHz}$, DMSO-d $): \delta 11.95$ (brs, $1 \mathrm{H}$ ), 8.72 (brs,1H), 8.46 (brs, $1 \mathrm{H}$ ), 8.09 (brs, $1 \mathrm{H})$, 7.95-7.93 (m, 4H), 7.81 (brs, $2 \mathrm{H}), 7.62$ (d, J = $11.2 \mathrm{~Hz}, 2 \mathrm{H}$ ), 7.18 (brs, 1H), 5.71 (brs, 2H), 3.90 (s, 3H), 3.80 (s, 3H); ESI-MS: m/z, $477.0(M+1)$.

(E)-N'-(4-(1-((3,4-dimethoxypyridin-2-yl) methyl)-1H-1,2,3-triazol-4-yl)benzylidene)-3,5dichlorobenzohydrazide $8 \mathrm{i}$

Pale yellow solid; Yield: $88 \%$; M.p.: $136-$ $138^{\circ} \mathrm{C}$; IR (KBr): $\mathrm{U}_{\max } 3452,3149,3086,2947,2841$, 1670, 1586, 1563, 1490, 1433, 1381, 1351, 1301, $1271,1231,1176,1133,1100,1067,996,974,944$, $903,876,864,824,805,748,707,663,602,533,499$ $\mathrm{cm}^{-1} ; 1 \mathrm{H}$ NMR (400 MHz, DMSO-d $): \delta 12.04$ (brs, $1 \mathrm{H}), 8.68(\mathrm{brs}, 1 \mathrm{H}), 8.44$ (brs, $1 \mathrm{H}), 8.11-7.89(\mathrm{~m}, 6 \mathrm{H})$, $7.81(\mathrm{~d}, \mathrm{~J}=10.4 \mathrm{~Hz}, 2 \mathrm{H}), 7.16$ (brs, $1 \mathrm{H}$ ), 5.72 (brs, 2H), $3.90(\mathrm{~s}, 3 \mathrm{H}), 3.80(\mathrm{~s}, 3 \mathrm{H})$; ESI-MS: m/z, 511.0 $(\mathrm{M}+1)$.

(E)-N'-(4-(1-((3,4-dimethoxypyridin-2-yl) methyl)-1H-1,2,3-triazol-4-yl)benzylidene)-2,5difluorobenzohydrazide 8 j
Pale yellow solid; Yield: 88\%; M.p.: 140$141^{\circ} \mathrm{C}$; IR (KBr): $U_{\max } 3446,3307,3131,3071,3019$, 2974, 2943, 2839, 2598, 1663, 1606, 1585, 1546, $1490,1453,1424,1359,1301,1274,1229,1180$, 1128, 1073, 996, 971, 949, 906, 883, 822, 779, 751, $727,690,669,620,579,538,498 \mathrm{~cm}^{-1} ;{ }^{1} \mathrm{H}$ NMR (400 $\left.\left.\mathrm{MHz}, \mathrm{DMSO}-\mathrm{d}_{6}\right): \delta 12.11{ }^{*} 11.96, \mathrm{~s}, 1 \mathrm{H}\right), 8.66\left({ }^{*} 8.58\right.$, $\mathrm{s}, 1 \mathrm{H}), 8.32\left({ }^{*} 8.09, \mathrm{~s}, 1 \mathrm{H}\right), 8.20(\mathrm{brs}, 1 \mathrm{H}), 7.97(\mathrm{~d}, \mathrm{~J}=$ $10.2 \mathrm{~Hz}, 2 \mathrm{H}), 7.87-7.70(\mathrm{~m}, 2 \mathrm{H}), 7.56-7.39(\mathrm{~m}, 3 \mathrm{H})$, 5.72 (brs, 2H), $3.90(\mathrm{~s}, 3 \mathrm{H}), 3.80(\mathrm{~s}, 3 \mathrm{H})$; ESI-MS: $\mathrm{m} / \mathrm{z}, 479.0(\mathrm{M}+1)$.

\section{Antibacterial and antifungal bioassay}

The antibacterial activity of all the hydrazone derivatives (8a-j) were examined against a panel of bacterial culture viz., Staphylococcus.pyogens, Staphylococcus.aureus (Gram positive) and Escherichia.coli, Pseudomonas.aeruginosa (Gram negative). The antibacterial activity was performed by agar well diffusion method with reference to Ciprofloxacin $(250 \mu \mathrm{g} / \mathrm{mL})$ as standard drug. Nutrient agar was used as culture media and DMSO was used as solvent control ${ }^{36-38}$. Similarly, the antifungal activity was evaluated Aspergillus niger and Candida albicans with reference to Nystatin as the standard antifungal drug. Sabouraud dextrose agar was used as culture media and DMF was used as solvent control ${ }^{39}$. The results of the antibacterial and antifungal activity was determined by measuring zone of inhibition and is tabulated Table 1.

\section{CONCLUSION}

In summary, the present paper describes the synthesis of some new 1,2,3-triazole-benzohydrazides 8a-j in six steps involving condensation of the key intermediate 4-(1-((3,4-dimethoxypyridin-2-yl) methyl)-1H-1,2,3-triazol-4-yl)benzaldehyde 6 with various benzohydrazide $7 a-j$ in quatitative yields. The antifungal screening of these hydrazones revealed that compounds $\mathbf{8 b}, \mathbf{8 d}, \mathbf{8 i}$ and $\mathbf{8 j}$ with $\mathrm{R}=4-\mathrm{OH}$, 4- $\mathrm{SO}_{2} \mathrm{Me}, 3,5$-dichloro and 2,5-difluoro substitution exhibited very good fungal activity and the remaining hydrazone derivatives in the series showed moderate antifungal activity.

\section{ACKNOWLEDGEMENTS}

The authors are grateful to JNTUH for providing facilities to carry out the work 


\section{REFERENCES}

1. Kaval, N.; Ermolat'ev, D.; Appukkuttan, P.; Dehaen, W.; Kappe, C. O.; Van der Eycken, E. J. Comb. Chem. 2005, 7, 490.

2. Hartmuth, C. K.; Barry, S. Drug Discovery Today. 2003, 8, 1128.

3. Yi, Xu.;Yan, W.; Lan, Y.; Rong-Mei, L.; Bao-Di, D.; Ren-Jie, T.; Ping-Hui, G.; Yuan-Ying, J. J. Proteome Res. 2009, 8, 5296.

4. Pasqualotto, A. C.; Denning, D. W. J. Antimicrob. Chemother. 2008, 61, i19- i30.

5. Alexander, A.; Tom, P.; Caremans, Jan, V.; Guy Van den, M.; Johan, A. M.; Patrick, A. Molecular Pharmaceutics. 2010, 7, 905.

6. Smith, J.; Safdar, N.; Knasinski, V.; Simmons, W.; Bhavnani, S. M.; Ambrose, P. G.; Andes, D. Voriconazole Antimicrob. Agents Chemother. 2006, 50, 1570.

7. Geria, A. N.; Scheinfeld, N. S. Drugs. 2008, 11, 661.

8. Schiller, D. S.; Fung, H. B. Clin. Ther. 2007, 29, 1862.

9. Xu, X. M.; Nicholson, P.; Ritieni, A. Int. J. Food Microbio. 2007, $119,67$.

10. Clark, T.; Deas, A. H. B. J. Chromatogr. 1985, 329, 181.

11. Toribio, L.; del Nozal, M. J.; Bernal, J. L.; Jiménez, J.J.; Alonso, C. J. Chromatography. A. 2004, 1046, 249.

12. Terence Grayson, B.; Sarah, L. B.; Alan, J. S.; Jeremy, N. D.; David, W. Pesticide Science. 1995, 45, 153.

13. Fuller, M. S.; Robertson, R. W.; Gisi, U. Pestic. Biochem. Physiol.1990, 36, 115.

14. Johannes, Kaulen. Angewandte. Chemie. Int. Ed. 1989, 462.

15. Eckert, M. R.; Rossall, S.; Selley, A.; Fitt, B. D. Pest. Management Science. 2010, 66, 396.

16. Malgorzata, B.; Zlatko, Z. Plant Growth Regulation. 2000, 30, 117.

17. Lazrek, H. B.; Taourirte, M.; Oulih, T.; Barascut, J. L.; Imbach, J. L.; Pannecouque, C.; Witrouw, M.; De Clercq, E. Nucleosides Nucleotides Nucleic Acids. 2001, 20, 1949.

18. Buckle, D. R.; Rockell, C. J.; Smith, H.; Spicer, B. A. J. Med. Chem. 1984, 27, 223.

19. De las Heras, F. G.; Alonso, R.; Alonso, G. J. Med. Chem. 1979, 22, 496.

20. El-Etrawy, A. Sh.; Abdel-Rahman, A. A.-H.
Heterocycl. Comp. 2010, 46, 1105.

21. Erhan, P.; Gülay, Pelin, K.; Tuba, D.; Gülçin, A. Farmaco. 2002, 57, 101.

22. Poulsen, S. A.; Wilkinson, B. L.; Innocenti, A Bioorg. Med. Chem. Lett. 2008, 18, 4624.

23. Hussain D.F. Orient J Chem. 2016, 32, 539.

24. Fraga, C.A.M.; Barreiro, E. J. Curr. Med. Chem. 2006, 13, 167.

25. Rollas, S.; Küçükgüzel, S.G. Molecules. 2007, 12, 1910.

26. Zulkepli, N.A.; Rou, K.V.K.; Sulaiman, W.N.H.W.; Salhin, A.; Saad, B.; Seeni, A. Asian Pac. J. Cancer Prev. 2011, 12, 259.

27. Wardakhan,W.W.; El-Sayed, N.N.R.; Mohareb, M. Acta Pharm. 2013, 63, 45.

28. Mickeviius, V.; Voskien, A.; Jonuškien, I.; Kolosej, R.; Šiugždait, J.; Venskutonis, P.R.; Kazernaviikt, R.; Brazien, Z.; Jakien, E. Molecules. 2013, 18, 15000.

29. Tumosien, I.; Jakien, E.; Kantminien, K.; Rutkauskas, K.; Beresneviius, Z.J. CHEMIJA. 2010, 21, 139.

30. Rice, L.B. Unmet medical needs in antibacterial therapy. Biochem. Pharmacol. 2006, 71, 991.

31. Kolb, H.C.; Finn, M.G.; Sharpless. K.B. Angew. Chem. 2001, 40, 2004.

32. Rostovtsev, V.V.; Green, L.G.; Fokin, V.V.; Sharpless, K.B. Angew. Chem. 2002, 114, 2708.

33. C.W. Tornoe, C. Christensen, M. Meldal. J. Org. Chem. 2002, 67, 3057.

34. Chawla, G.; Kaushik, D.; Khan, S.A.; Suresh. K. Eur.J.Med. Chem. 2010, 45, 3943.

35. Jian-Zhong, C.; Shuang, H.; Xie, X.; Fei-Fei, Z. Eur.J. Med. Chem. 2014, 74, 73.

36. Daniela Batovska, StoyanParushev, BistraStamboliyska, Iva Tsvetkova, Mariana Ninova, HristoNajdenski. Eur.J.Med.Chem. 2009, 44, 2211.

37. Tomar, V.; Bhattacharjee, G.; Kamaluddin.;Ashok Kumar. Bioorg. Med. Chem. Lett. 2007, 17, 5321.

38. Rajendra Prasad Y.; Lakshmana Rao A.; Rambabu R. E.J.Chem. 2008, 5, 461.

39. Lahtchev, K. L.; Batovska, D. I.; St. Parushev, P.; Ubiyvovk, V. M.; Sibirny, A.A. Eur. J.Med. Chem. 2008, 43, 220. 